\title{
G6b-B regulates an essential step in megakaryocyte maturation
}

\section{Short title:}

\section{MK maturation defect in G6b-B-null mice}

Isabelle C. Becker, ${ }^{1,2 \dagger}$ Zoltan Nagy, ${ }^{1,2 \dagger}$ Georgi Manukjan, ${ }^{1}$ Melanie Haffner-Luntzer, ${ }^{3}$ Maximilian Englert, ${ }^{1,2}$ Tobias Heib,, ${ }^{1,2}$ Timo Vögtle, ${ }^{1,2}$ Carina Gross, ${ }^{1,2}$ Richa Bharti, ${ }^{4}$ Sascha Dietrich, ${ }^{4}$ Kristina Mott, ${ }^{1}$ Johannes Heck, ${ }^{1}$ Anke Jeschke, ${ }^{5}$ Thorsten Schinke, ${ }^{5}$ Nicolas Schlegel, ${ }^{6}$ Tobias Heckel, ${ }^{4}$ David Stegner, ${ }^{1,2}$ Irina Pleines, ${ }^{1,2}$ Anita Ignatius, ${ }^{3}$ Harald Schulze, ${ }^{1 *}$ and Bernhard Nieswandt ${ }^{1,2^{*}}$

${ }^{1}$ Institute of Experimental Biomedicine, University Hospital, University of Würzburg, Würzburg, Germany.

${ }^{2}$ Rudolf Virchow Center for Integrative and Translational Bioimaging, University of Würzburg, Würzburg, Germany.

${ }^{3}$ Institute of Orthopaedic Research and Biomechanics, University Medical Center UIm, UIm, Germany.

${ }^{4}$ Core Unit Systems Medicine, University of Würzburg, Würzburg, Germany.

${ }^{5}$ Department of Osteology and Biomechanics, University Medical Center Hamburg-Eppendorf, Hamburg, Germany.

${ }^{6}$ Department of General Visceral, Vascular and Paediatric Surgery (Department of Surgery I), University of Würzburg, Würzburg, Germany

$\dagger, *$ Authors contributed equally.

¥Current affiliation: Vascular Biology Program, Boston Children’s Hospital and Harvard Medical School, Boston, MA 02115

"Correspondence to: Bernhard Nieswandt, PhD; E-mail: bernhard.nieswandt@virchow.uniwuerzburg.de or Harald Schulze, PhD; E-mail: harald.schulze@uni-wuerzburg.de

Text word count: 1200

Abstract word count: 200

Number of figures: 2 figures

Number of supplementary files: 4 supplementary figures, 1 supplementary table, 3 movies

Number of references: 25 


\begin{abstract}
G6b-B is a megakaryocyte lineage-specific immunoreceptor tyrosine-based inhibition motif (ITIM)-containing receptor, essential for platelet homeostasis. Mice with a genomic deletion of the entire Mpig6b locus develop severe macrothrombocytopenia and myelofibrosis, which is reflected in humans with null-mutations in MPIG6B. The current model proposes that megakaryocytes lacking G6b-B develop normally, while proplatelet release is hampered, but the underlying molecular mechanism remains unclear. Here, we report on a spontaneous recessive single nucleotide mutation in C57BL/6 mice, localized within the intronic region of the Mpig6b locus that abolishes G6b-B expression and reproduces macrothrombocytopenia, myelofibrosis and osteosclerosis. As the mutation is based on a single nucleotide exchange, Mpig $6 b^{m u t}$ mice represent an ideal model to study the role of G6b-B. Megakaryocytes from these mice were smaller in size, displayed a less developed demarcation membrane system and reduced expression of receptors. RNA sequencing revealed a striking global reduction in the level of megakaryocyte-specific transcripts, in conjunction with decreased protein levels of the transcription factor GATA-1, and impaired thrombopoietin signaling. The reduced number of mature MKs in the bone marrow was corroborated on a newly developed Mpig6 $b$ null mouse strain. Our findings highlight an unexpected essential role of G6b-B in the early differentiation within the megakaryocytic lineage.
\end{abstract}




\section{Introduction}

Megakaryocytes (MKs) are large, polyploid cells within the bone marrow (BM) that develop in a complex differentiation process. Mature MKs harbor granules and internal membrane systems, which are indispensable for the assembly and release of functional platelets. ${ }^{1}$ G6b-B has been identified as an essential regulator of platelet biogenesis.. ${ }^{2}$ Deletion of the Mpig6b locus in mice results in macrothrombocytopenia and myelofibrosis, ${ }^{2}$ which was recently recapitulated in humans with disease-causing null-variants within MPIG6B. ${ }^{3-7}$ It was hypothesized that G6b-B affects a terminal step in platelet production, ${ }^{2,8}$ however, mechanistic insights are yet lacking. By characterizing a spontaneous Mpig6b mutant together with a newly generated Mpig6b-null mouse line we provide unexpected experimental evidence that establishes G6b-B as a central regulator for MK maturation, which can explain the complex phenotype of these mice.

\section{Methods}

Homozygous mutant mice carrying a spontaneously developed mutation in a splice acceptor site of Mpig6b (NM_001033221.3; c.404-1G>A) are referred to as Mpig6b ${ }^{\text {mut }}$. All methods are described in the supplementary methods. Whole exome sequencing data was submitted to NCBI SRA (BioProject-ID: PRJNA655378). RNA sequencing data are available at NCBI GEO (accession number: GSE155735).

\section{Results and Discussion}

\section{Single nucleotide exchange in Mpig6b results in macrothrombocytopenia}

In a breeding colony of C57BL/6 mice we identified individual animals with bleedings due to severe macrothrombocytopenia (Figure 1a-b). 10 generations of backcrossing led to isolation of a substrain presenting with a recessive trait. Using whole exome sequencing, we identified a homozygous single nucleotide exchange within Mpig6b (c.404-1G>A) in 10/10 mice (allele frequency: 1.0), resulting in abolished G6b-B protein expression in platelets (Figure 1c-e, S1ab). In silico prediction unraveled the introduction of a splice acceptor site in intron 2-3 of Mpig6b leading to an out-of-frame shift transcript with multiple stop codons, expected to result in nonsense-mediated mRNA decay. Absence of G6b-B led to a reduction in surface expression levels of platelet membrane glycoproteins (GPs) (Table S1), infinite tail bleeding times, myelofibrosis, splenomegaly and additional osteosclerosis in female Mpig6 $b^{\text {mut }}$ mice (Figure S1c-f). We thus identified a spontaneous single nucleotide mutation within Mpig6b resulting in a phenotype, which faithfully recapitulates $M p i g 6 b^{-/}$and $M p i g 6 b$ diY/F mice..$^{2,8,9}$ 
Analyzing BM-derived MKs from Mpig6 $b^{m u t}$ mice in vitro, we found that the number of proplatelet-forming cells was significantly reduced compared to the wildtype (WT) (Figure S2ad). The cytoskeleton of proplatelet-forming $M p i g 6 b^{\text {mut }}$ MKs, however, appeared morphologically comparable. Intravital two-photon microscopy on the cranial BM revealed a high degree of MK fragmentation together with a low amount of circulating platelets in Mpig $6 b^{\text {mut }}$ mice (movie S1-3).

\section{Impaired maturation of Mpig6 $\mathbf{b}^{\text {mut }}$ MKs}

The diameter of in vitro-matured Mpig $6 b^{\text {mut }}$ MKs was significantly smaller (Figure 1f). When native BM cells were analyzed flow cytometrically, we observed an increased percentage of MKs in Mpig6 $6 b^{\text {mut }}$ mice, also displaying a smaller size (Figure 1g-h). Transmission electron microscopy (TEM) analysis of BM MKs in situ revealed severely defective maturation of the demarcation membrane system (DMS) in Mpig6 $b^{\text {mut }}$ mice (Figure 2i-k). We also observed increased neutrophil emperipolesis into mutant MKs in situ by TEM and in cryosections (Figure S2e-f). Ploidy analysis showed a significant rise in $2 n$ megakaryoblasts, while the fractions of $16 n$ and $32 n$ MKs were marginally reduced (Figure S2g-h). Interestingly, the percentage of small- and medium-sized MKs was markedly increased, whereas the fraction of big and highly granular, side scatter high (SSC ${ }^{\text {high }}$ ) MKs was severely reduced in the BM of Mpig6 $b^{\text {mut }}$ mice (Figure 2a-b). The expression levels of prominent GPs positively correlated with MK size in WT animals, in which SSC ${ }^{\text {high }}$ MKs exhibited the highest GP surface abundance (Figure S3a). Notably, the overall GP surface expression in the entire MK population revealed a marked reduction for all GPs in Mpig6 $b^{\text {mut }}$ mice (Figure 2c), due to the accumulation of immature MKs expressing lower levels of the respective GPs. These findings were recapitulated in in vitromatured MKs (Figure S3b-c). The absence of a major block in MK ploidy levels together with reduced surface expression of GPlba, $\alpha 2$ integrin and GPVI are coherent with data from

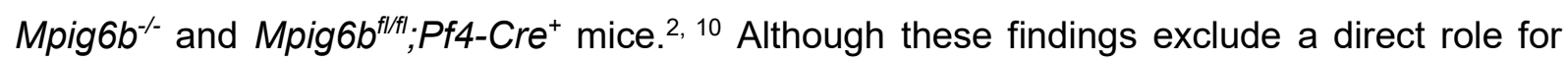
G6b-B in endomitosis, they point to a central function in regulating MK maturation. Interestingly, this resembles other mouse lines, where reduced MK maturation is associated with normal ploidy, including $\mathrm{Gfi}_{1} \mathrm{~b}^{-/ 11}, \mathrm{Nfe} 2^{-/-}$mice $^{12}$, or Rhoa ${ }^{f / f / l} ; \mathrm{Cdc} 42^{f / f / t} ; \mathrm{Pf} 4-\mathrm{Cre}^{+}$mice. ${ }^{13}$ The accumulation of immature MKs can lead to myelofibrosis, osteosclerosis, or both, as reported for Gata-1 ${ }^{\text {low }}$ or $N f e 2^{-/}$mouse lines, ${ }^{14-16}$ and might explain the phenotypes in G6b-B null mice.

\section{Defective MK-specific gene expression in Mpig $6 b^{m u t}$ mice}

Next, we performed bulk RNA sequencing on purified, native BM MKs from young adult mice, not yet displaying myelofibrosis. The transcriptome signature of Mpig6 $6 b^{\text {mut }} \mathrm{MKs}$ revealed a striking decrease in a plethora of MK-specific transcripts, including Tubb1, Myl9 or Gp1ba (Figure 2d). mRNA levels of transcription factors GATA-1and Gfi1b, which are indispensable for MK differentiation, ${ }^{17,}{ }^{18}$ were significantly less abundant in Mpig6 $b^{\text {mut }}$ MKs (Figure 2e). 
qPCR analysis revealed that transcript levels of Itgb3, Tubb1 and Gata1 were also significantly reduced when mutant MKs were in vitro-differentiated (Figure S3d). In addition, GATA1 protein levels were reduced in both native MKs in situ (Figure 2f), as well as in in vitrodifferentiated Mpig6 $b^{\text {mut }}$ MKs (Figure 2g, S3e). Our RNA-sequencing data strongly implies that loss of G6b-B hampers early transcriptional pathways within the megakaryocytic lineage, as reflected by downregulation of many MK-specific genes in primary MKs.

\section{Defective TPO-signaling in Mpig6 ${ }^{\text {mut }}$ MKs}

Mpig6 $b^{\text {mut }}$ mice exhibited elevated thrombopoietin (TPO) plasma levels (Figure S3e), as expected in response to low platelet counts. We thus investigated the total MK protein and phosphorylation levels of proteins, which are crucial to TPO signaling, ${ }^{10,19}$ Protein levels of cMpl, Jak2 and Shp2 were decreased in Mpig6 $b^{\text {mut }}$ MKs compared to WT MKs, while STAT5a/b were not significantly altered. Moreover, c-Mpl, Jak2, STAT5a/b and Shp2 phosphorylation was markedly reduced (Figure $\mathbf{2 g}$, S3f). Our findings thus provide evidence that loss of G6bB disturbs TPO-based signaling events in MKs and point to a critical role of G6b-B in regulating relevant signaling pathways driving early MK maturation.

\section{Mpig6 $6 b^{-/}$mice display less mature MKs}

To corroborate that our findings reflect the role of G6b-B and are not the consequence of an unlikely co-segregating mutation, we generated a novel Mpig6 $6 b^{-/-}$mouse model (Figure S4ad). MKs from this novel Mpig $6 \mathrm{~b}^{-/}$mice displayed an increase in the percentage of small- and medium-sized MKs and a marked decrease in big and SSC ${ }^{\text {high }}$ MKs (Figure 2h), thus confirming that lack of G6b-B results in a MK maturation defect.

Patients with disease-causing variants within MPIG6B present(ed) with congenital macrothrombocytopenia, mild-to-moderate bleeding diathesis, focal myelofibrosis and atypical MKs, however the underlying causes of the disease have remained unclear. ${ }^{3-7}$ Our results demonstrate a previously unrecognized key function of G6b-B in MK maturation by regulating cell size, DMS development, receptor levels and gene expression. These findings thus challenge the previous concept, which proposed a rather unaffected development of G6b-B deficient $\mathrm{MKs}^{2}, 4,8,20$, and provide an unexpected mechanistic explanation for the severe macrothrombocytopenia in Mpig $6 b^{\text {mut }}$ mice, as a direct consequence of an overall MK maturation defect. We observed an increased number of immature MKs in the bone marrow, which might help to better understand the cause of myelofibrosis in G6b-B null mice and patients, as an accumulation of immature MKs has been described as the main driver of this complex disease. ${ }^{21-25}$ 


\section{Acknowledgements}

We thank Stefanie Hartmann, Sylvia Hengst, Daniela Naumann and Mariola Dragan for excellent technical assistance and the microscopy platform of the Bioimaging Center Würzburg for providing technical infrastructure and support. We thank Prof. Dr. Yotis Senis (Strasbourg, France) for providing anti-G6b-B antibodies. We also thank the Core Unit SysMed at the University of Würzburg for excellent technical support and RNA-seq data generation.

\section{Funding}

Deutsche Forschungsgemeinschaft (DFG, German Research Foundation) (Project number 374031971 - TRR 240/project A01 to BN and project A03 to HS, and grant NI 556/11-2 to BN)

IZKF at the University of Würzburg (project Z-6)

European Union (EFRE - Europäischer Fonds für regionale Entwicklung, Bavaria)

German Excellence Initiative to the Graduate School of Life Sciences, University of Würzburg (to IB and ZN)

\section{Author contributions}

Conceptualization: ICB, ZN,GM, HS, BN; Methodology: MHL, RB, SD; Software: TH, RB, SD; Investigation: ICB, ZN, GM, MHL, ME, TH, TV, CG, JH, AJ; Resources: TS, NS, TH, DS, HS, BN; Supervision: IP, AI, HS, BN; Writing - original draft: ICB, ZN, GM, HS, BN; Writingreview \& editing: ICB, ZN, GM, MHL, IP, HS, BN

\section{Declaration of Interests}

The authors declare no competing interests.

\section{Data availability}

Whole exome sequencing data is available under the BioProject-ID PRJNA655378 (https://www.ncbi.nlm.nih.gov/bioproject/). Sequencing data are available at NCBI GEO (http://www.ncbi.nlm.nih.gov/geo) under the accession number GSE155735. 


\section{References}

1. Machlus KR, Italiano JE, Jr. The incredible journey: From megakaryocyte development to platelet formation. J Cell Biol. 2013;201(6):785-796.

2. Mazharian A, Wang YJ, Mori J, et al. Mice lacking the ITIM-containing receptor G6b-B exhibit macrothrombocytopenia and aberrant platelet function. Sci Signal. 2012;5(248):ra78.

3. Melhem M, Abu-Farha M, Antony D, et al. Novel G6B gene variant causes familial autosomal recessive thrombocytopenia and anemia. Eur J Haematol. 2017;98(3):218-227.

4. Hofmann I, Geer MJ, Vogtle T, et al. Congenital macrothrombocytopenia with focal myelofibrosis due to mutations in human G6b-B is rescued in humanized mice. Blood. 2018;132(13):1399-1412.

5. Saliba AN, Ferrer A, Gangat N, et al. Aetiology and outcomes of secondary myelofibrosis occurring in the context of inherited platelet disorders: A single institutional study of four patients. Br J Haematol. 2020;

6. Chen $\mathrm{H}$, Zheng J, Chen Z, Ma H, Zhang R, Wu R. Case report of a novel MPIG6B gene mutation in a Chinese boy with pancytopenia and splenomegaly. Gene. 2019;715(143957.

7. Batis $\mathrm{H}$, Almugairi $\mathrm{A}$, Almugren $\mathrm{O}$, et al. Detrimental variants in MPIG6B in two children with myelofibrosis: Does immune dysregulation contribute to myelofibrosis? Pediatr Blood Cancer. 2021;e29062.

8. Stavnichuk M, Tauer JT, Nagy Z, et al. Severity of megakaryocyte-driven osteosclerosis in Mpig6b-deficient mice is sex-linked. J Bone Miner Res. 2021;

9. Geer MJ, van Geffen JP, Gopalasingam P, et al. Uncoupling ITIM receptor G6b-B from tyrosine phosphatases Shp1 and Shp2 disrupts murine platelet homeostasis. Blood. 2018;132(13):1413-1425.

10. Mazharian A, Mori J, Wang YJ, et al. Megakaryocyte-specific deletion of the proteintyrosine phosphatases Shp1 and Shp2 causes abnormal megakaryocyte development, platelet production, and function. Blood. 2013;121(20):4205-4220.

11. Foudi A, Kramer DJ, Qin J, et al. Distinct, strict requirements for Gfi-1b in adult bone marrow red cell and platelet generation. J Exp Med. 2014;211(5):909-927.

12. Shivdasani RA, Rosenblatt MF, Zucker-Franklin D, et al. Transcription factor NF-E2 is required for platelet formation independent of the actions of thrombopoietin/MGDF in megakaryocyte development. Cell. 1995;81(5):695-704.

13. Heib T, Hermanns HM, Manukjan G, et al. RhoA/Cdc42 signaling drives cytoplasmic maturation but not endomitosis in megakaryocytes. Cell Rep. 2021;35(6):109102.

14. Shivdasani RA, Fujiwara $Y$, McDevitt MA, Orkin SH. A lineage-selective knockout establishes the critical role of transcription factor GATA-1 in megakaryocyte growth and platelet development. EMBO J. 1997;16(13):3965-3973.

15. Vannucchi AM, Bianchi L, Paoletti F, Di Giacomo V, Migliaccio G, Migliaccio AR. Impaired GATA-1 expression and myelofibrosis in an animal model. Pathol Biol (Paris). 2004;52(5):275-279.

16. Kacena MA, Shivdasani RA, Wilson K, et al. Megakaryocyte-osteoblast interaction revealed in mice deficient in transcription factors GATA-1 and NF-E2. J Bone Miner Res. 2004;19(4):652-660.

17. Tijssen MR, Ghevaert C. Transcription factors in late megakaryopoiesis and related platelet disorders. J Thromb Haemost. 2013;11(4):593-604.

18. Schulze H, Shivdasani RA. Mechanisms of thrombopoiesis. J Thromb Haemost. 2005;3(8):1717-1724.

19. Miyakawa $\mathrm{Y}$, Rojnuckarin $\mathrm{P}$, Habib T, Kaushansky K. Thrombopoietin induces phosphoinositol 3-kinase activation through SHP2, Gab, and insulin receptor substrate proteins in BAF3 cells and primary murine megakaryocytes. J Biol Chem. 2001;276(4):24942502. 
20. Vogtle T, Sharma S, Mori J, et al. Heparan sulfates are critical regulators of the inhibitory megakaryocyte-platelet receptor G6b-B. Elife. 2019;8(

21. Wen QJ, Yang Q, Goldenson B, et al. Targeting megakaryocytic-induced fibrosis in myeloproliferative neoplasms by AURKA inhibition. Nat Med. 2015;21(12):1473-1480.

22. Gangat N, Marinaccio C, Swords R, et al. Aurora Kinase A Inhibition Provides Clinical Benefit, Normalizes Megakaryocytes, and Reduces Bone Marrow Fibrosis in Patients with Myelofibrosis: A Phase I Trial. Clin Cancer Res. 2019;25(16):4898-4906.

23. Malara A, Abbonante V, Zingariello M, Migliaccio A, Balduini A. Megakaryocyte Contribution to Bone Marrow Fibrosis: many Arrows in the Quiver. Mediterr J Hematol Infect Dis. 2018;10(1):e2018068.

24. Vannucchi AM, Bianchi L, Cellai C, et al. Development of myelofibrosis in mice genetically impaired for GATA-1 expression (GATA-1(low) mice). Blood. 2002;100(4):11231132.

25. Melo-Cardenas J, Migliaccio AR, Crispino JD. The Role of Megakaryocytes in Myelofibrosis. Hematol Oncol Clin North Am. 2021;35(2):191-203. 


\section{Figure Legends}

Figure 1. Single nucleotide mutation within Mpig6b results in severe macrothrombocytopenia and impaired MK maturation. Platelet count (a) and volume (b) in 10-week-old female and male WT and Mpig6 $6 b^{\text {mut }}$ mice were assessed by an automated blood cell analyzer. Values are mean \pm SD. Unpaired, two-tailed Student's t-test. ${ }^{* *} P<0.001$. (c) Identification of a mutation in a splice acceptor site of $M p i g 6 b$ in $M p i g 6 b^{\text {mut }}$ mice by whole exome sequencing. The $\mathrm{G}>\mathrm{A}$ single nucleotide exchange in Mpig6b was present in all reads in mutant, but not in WT mice. (d-e) Absence of G6b-B was validated in Mpig6 $6 b^{\text {mut }}$ platelets by flow cytometry (d) and immunoblotting (C-terminal antibody) (e). (f) WT and Mpig6b ${ }^{\text {mut }} \mathrm{MKs}$ were matured in vitro in the presence of TPO and analyzed by brightfield microscopy. Mean MK diameter was determined manually using ImageJ software. At least 30 MKs per culture were analyzed. Values are mean $\pm S D(n=3)$. Unpaired, two-tailed Student's t-test. ${ }^{*} P<0.05$. (g) The allbß3-positive cell population in whole BM of $W T$ or Mpig $6 b^{\text {mut }}$ mice was analyzed by flow cytometry. Values are mean $\pm S D(n=8)$. Unpaired, two-tailed Student's t-test. ${ }^{* * *} P<$ 0.001. (h) Mean size of native MKs was analyzed ex vivo by flow cytometry. Values are mean $\pm \mathrm{SD}(\mathrm{n}=4)$. Unpaired, two-tailed Student's t-test. ${ }^{* * *} \mathrm{P}<0.001$. (i) Demarcation membrane system (DMS) maturation in WT and $M p i g 6 b^{\text {mut }}$ BM MKs was visualized by TEM. Scale bars: $3 \mu \mathrm{m}$; insets: $1.5 \mu \mathrm{m}$. Nuclear $(\mathbf{j})$ and DMS fraction $(\mathbf{k})$ in relation to cell size were quantified manually using Image J software. At least $7 \mathrm{MKs}$ per mouse were analyzed. Values are mean $\pm S D(n=3)$. Unpaired, two-tailed Student's t-test. ${ }^{*} P<0.05$; ${ }^{* *} P<0.001$.

Figure 2. Maturation block in Mpig6 $b^{\text {mut }}$ MKs involves reduced gene expression and TPO signaling. (a, b) Mean size distribution of $W T$ and Mpig $6 b^{m u t}$ MKs was analyzed by flow cytometry. (a) Dot plots depicting the proportion of SSC ${ }^{\text {high }}$ MKs and the delineation between small, medium and big MKs. (b) Values are mean \pm SD $(n=6)$. Unpaired, two-tailed Student's t-test. ${ }^{*} \mathrm{P}<0.05$; ${ }^{* *} \mathrm{P}<0.01$; ${ }^{* *} \mathrm{P}<0.001$. (c) Mean surface receptor expression on the whole MK population derived from $W T$ and Mpig6 $b^{\text {mut }}$ mice. Values are mean $\pm S D(n=4)$. Unpaired, two-tailed Student's t-test. ${ }^{* * *} \mathrm{P}<0.001$. (d) Volcano plot showing up- and downregulation of genes in native Mpig $6 b^{\text {mut }}$ MKs derived from female mice compared to female $W T$ controls. Black lines point towards downregulated MK-specific genes (e.g. Tubb1, Clec1b, Gp1bb). (e) Up- and downregulation of MK differentiation-related transcription factors in native MKs from male and female $M p i g 6 b^{\text {mut }}$ mice compared to the respective control $(n=4)$. Only values with a log2 fold change $<0.5849$ (dotted line) were considered downregulated. (f) Immunostainings of femora cryosections visualizing GATA-1 expression in $W T$ and Mpig $6 b^{\text {mut }}$ MKs in situ. Scale bars: $50 \mu \mathrm{m}$. Runx1: Runt-related transcriptions factor 1; Fli1: Friend leukemia integration 1 transcription factor; Etv6: ETS variant transcription factor 6; Fog1: Friend of GATA-1; Gfi1b: growth factor-independent $1 \mathrm{~B}$ transcriptional repressor. (g) Phosphorylation and/or total 
expression levels of GATA-1, c-Mpl, Jak2, STAT5a/b, Shp1 and Shp2 in TPO-stimulated WT and $M p i g 6 b^{\text {mut }}$ MKs were visualized using an automated quantitative capillary-based immunoassay platform; Jess (ProteinSimple). (h) Mean size distribution of WT and Mpig6 $b^{-/-}$ MKs was analyzed by flow cytometry. Values are mean \pm SD $(n=2)$. Unpaired, two-tailed Student's t-test. ${ }^{*} \mathrm{P}<0.05$. 

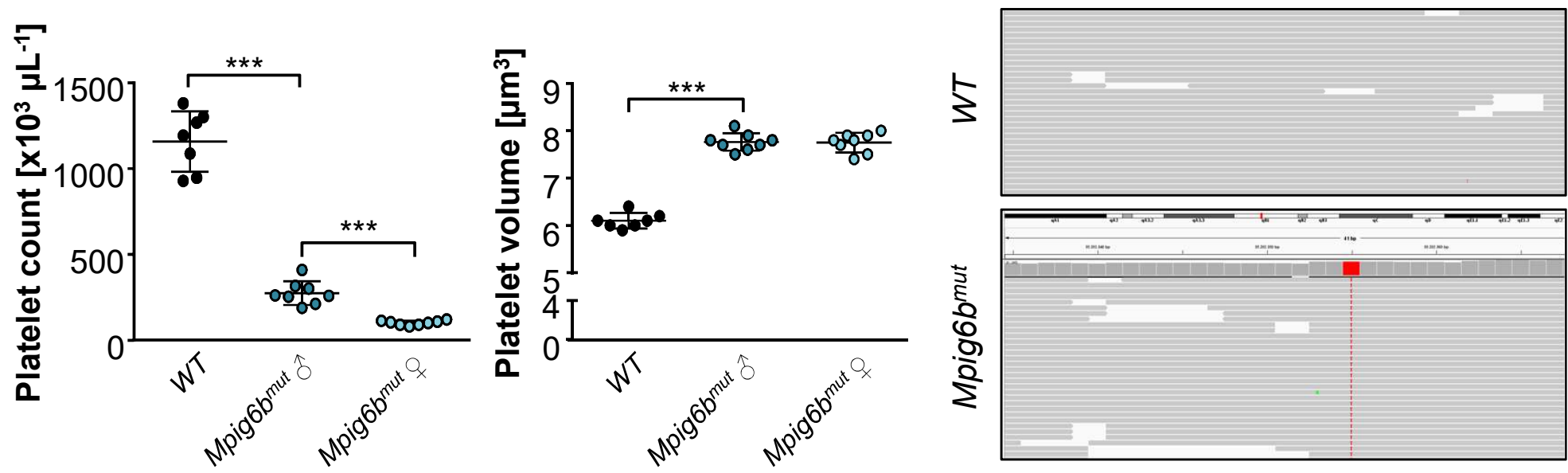

d

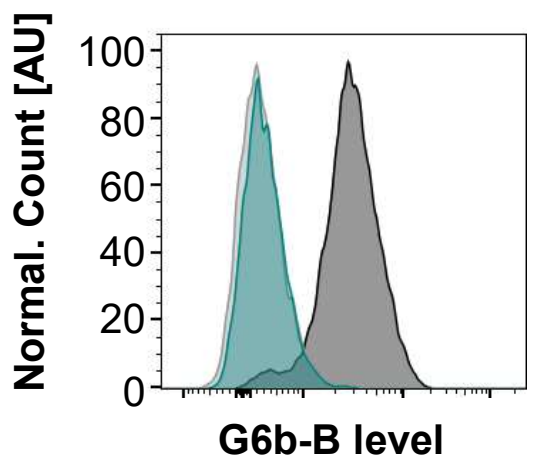

- $W T$ - secondary only

- WT

- Mpig6b ${ }^{\text {mut }}$

\section{g}

h
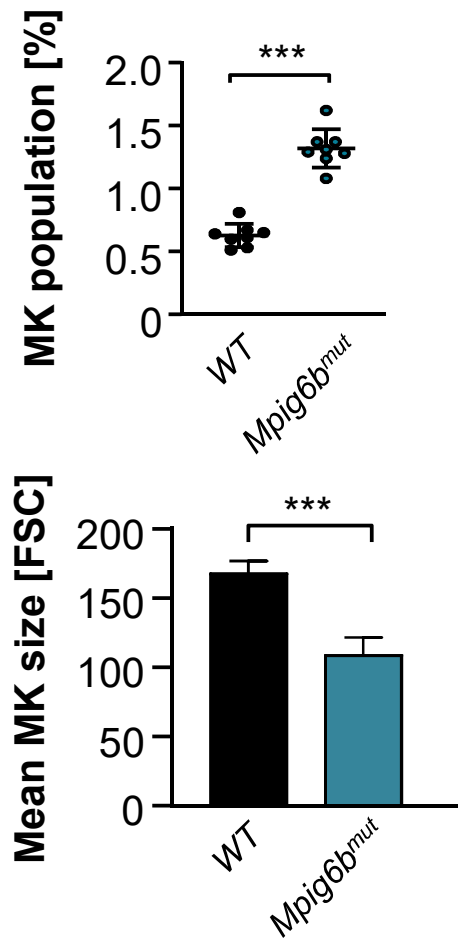

e

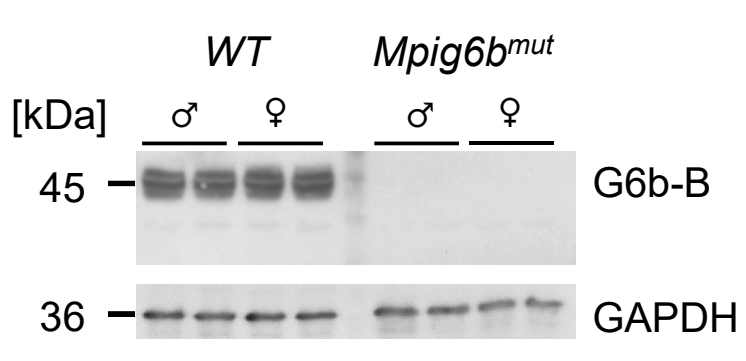

f

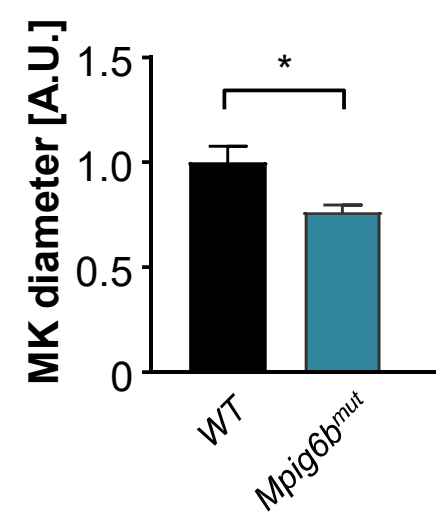

j

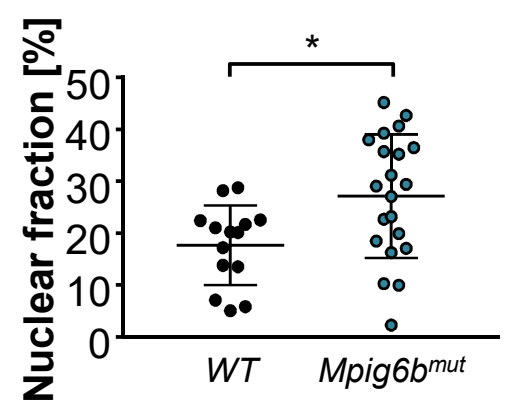

$k$

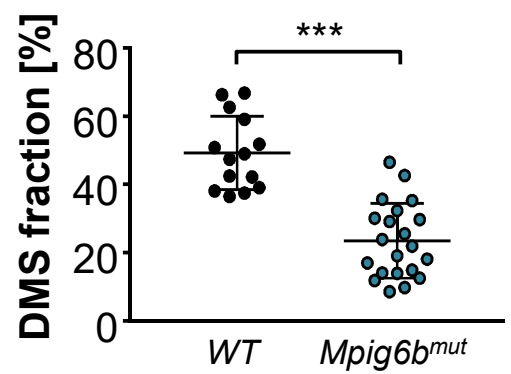


bioRxiv preprint doi: https://doi.org/10.1101/2021.11.11.468226; this version posted November 12, 2021. The copyright holder for this

a

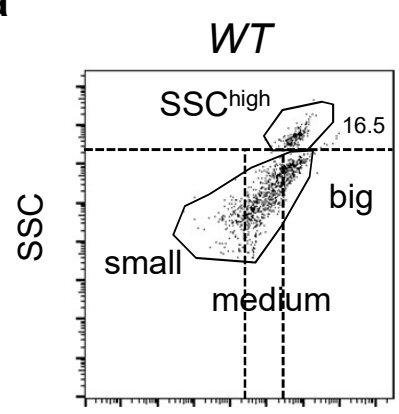
perpetuity. It is made available under bCC-BY-NC-ND 4.0 International license.
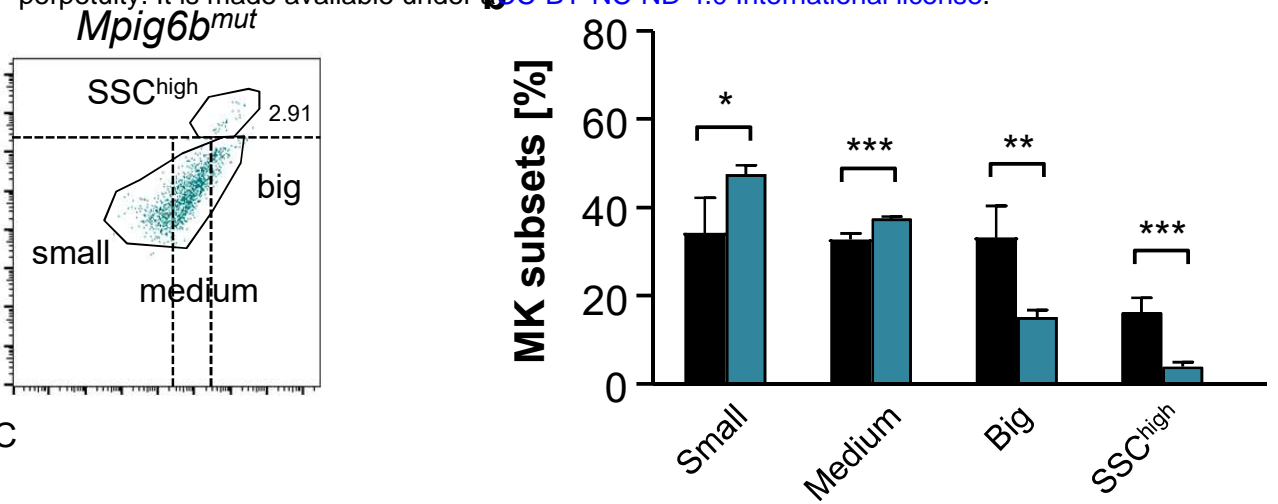

C
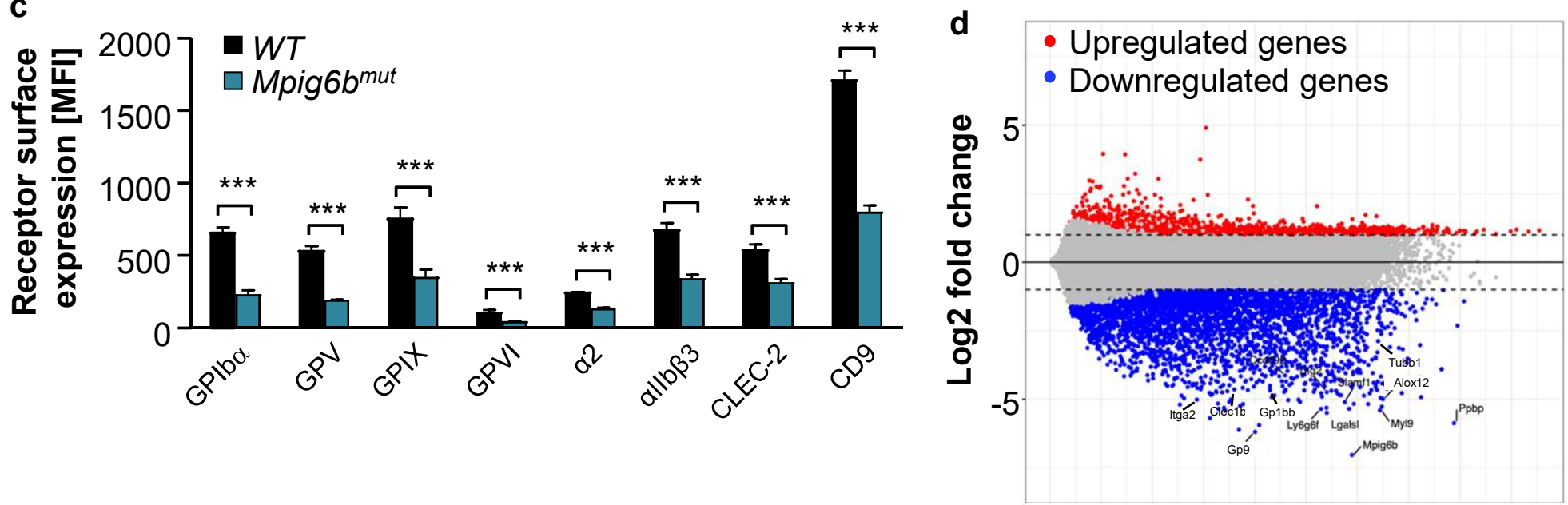

e

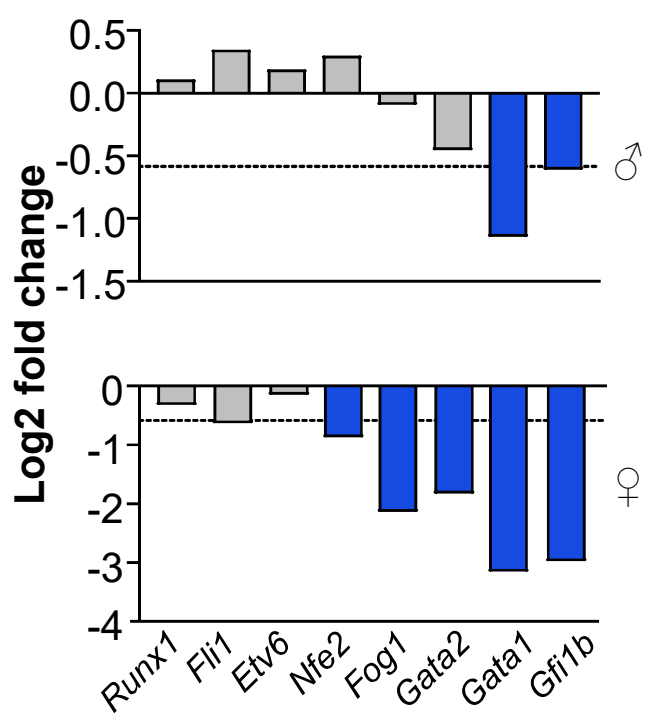

h

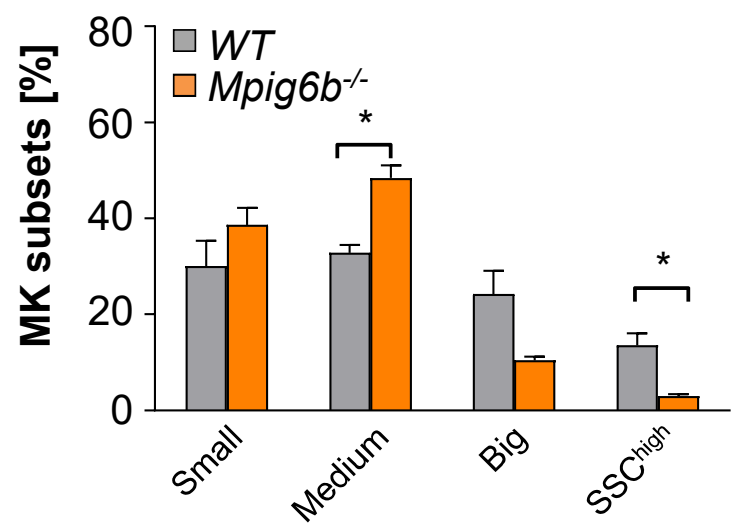

g

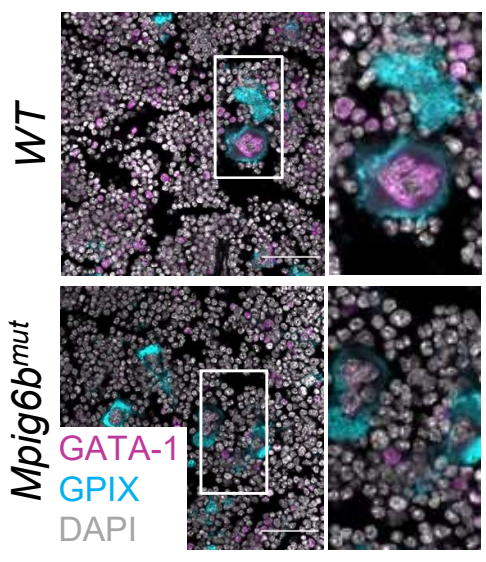

WT Mpig6b" ${ }^{\text {mut }}$ WT Mpig6 $6 b^{\text {mut }}$

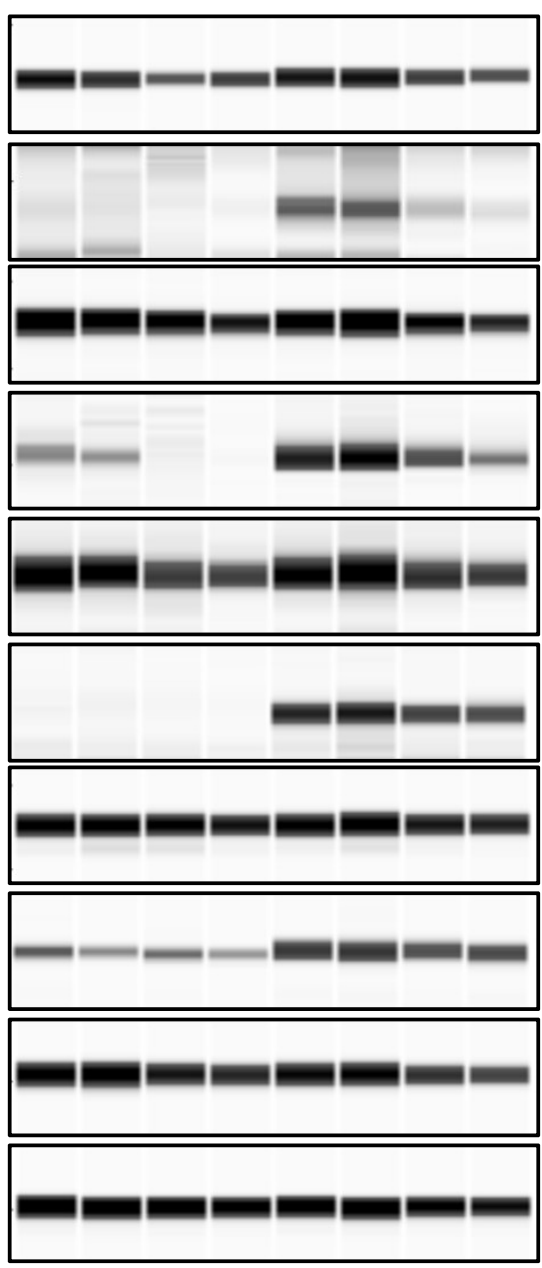

GATA-1

c-Mpl

p-Tyr626

c-Mpl

Jak2

p-Tyr1007/

1008

Jak2

STAT5a/b

p-Tyr694/

699

STAT5a/b

Shp2

p-Tyr580

Shp2

Shp1 